\title{
Atendimento odontológico de pacientes surdo-cegos: enfrentando desafios
}

\author{
Dental care for deafblind patients: facing challenges
}

Antônio Augusto Iponema Costa*

Alvaro Della Bona**

\section{Resumo}

Introdução: a odontologia tem um papel muito importante na atenção à saúde dos pacientes surdo-cegos, porém, esbarra na falta de profissionais qualificados e estudos específicos nessa área. Objetivo: essa revisão de literatura tem por objetivo apresentar aos profissionais da odontologia informações sobre a surdo-cegueira, descrevendo conceitos, causas e classificações, assim como relatar os métodos de comunicação, a necessidade da inserção do odontólogo na equipe multiprofissional de atendimento aos surdo-cegos, definindo, por fim, metodologias que auxiliem no atendimento odontológico desses pacientes. Revisão de literatura: a atuação do cirurgião-dentista na equipe multiprofissional proporciona aos pacientes melhorias no seu desenvolvimento, por meio da criação de vínculos e de atividades que propiciem maior autonomia. Além disso, demonstra a deficiência de informação na área da surdo-cegueira, sendo o envolvimento da família fator fundamental para um prognóstico favorável no processo educacional, social e de comunicação. Considerações finais: o profissional, além do conhecimento técnico e científico em sua área de atuação, deve ter domínio do melhor meio de comunicação com seu paciente surdo-cego.

Palavras-chave: Surdo-cegueira. Comunicação interdisciplinar. Pessoas com deficiência. Barreiras de comunicação.

\section{Introdução}

A partir de 2001, com a Resolução 22/2001 do Conselho Federal de Odontologia (CFO) ${ }^{1}$, foi homologada, como especialidade, a Odontologia para Pacientes com Necessidades Especiais. Os profissionais dessa especialidade estão aptos ao atendimento dos diferentes tipos de comprometimento (físico, intelectual, sensorial, orgânico, social e/ou comportamental). Dentre esses pacientes considerados "especiais", encontram-se os deficientes sensoriais, bem como os surdo-cegos.

A surdo-cegueira baseia-se no acometimento dos sentidos sensoriais da visão e da audição, podendo ter diferentes níveis de gravidade. A deficiência auditiva e visual provoca consequências no desenvolvimento infantil que irão depender do grau e do período de surgimento ${ }^{2}$. Conforme o Bureau International of Audiophonologie (BIAP), a deficiência auditiva é classificada em leve (intervalo de 20 a $40 \mathrm{~dB}$ ), moderada (intervalo de 40 a $70 \mathrm{~dB}$ ), severa (intervalo de 70 a $90 \mathrm{~dB}$ ) e profunda (superior a $90 \mathrm{~dB}$ ). Considera-se normal a audição no intervalo de 10 a $20 \mathrm{~dB}$. As perdas visuais são caracterizadas pela incapacidade funcional total (cegueira) ou parcial (baixa visão) ${ }^{2}$.

A deficiência auditiva provoca, naturalmente, no indivíduo a necessidade de observação visual mais apurada. O deficiente visual, por sua vez, de-

Mestrando do Programa de Pós-Graduação em Odontologia da Universidade de Passo Fundo, Passo Fundo, RS, Brasil.

Professor titular do Programa de Pós-Graduação em Odontologia da Universidade de Passo Fundo, Passo Fundo, RS, Brasil. 
senvolve sentidos como estímulos táteis, olfativos, vestibulares e de propriocepções. Quando se fala de uma pessoa surdo-cega, não se deve pensar que se trata de um sujeito cego que não pode ouvir, ou de um surdo que não pode ver, mas de uma pessoa com limitações que interferem no desenvolvimento social, comportamental, de aprendizagem e adaptação ao meio. Não se está diante de uma simples somatória de deficiências visuais e auditivas ${ }^{3,4}$.

De acordo com dados estimados pela Organização Mundial de Saúde (OMS) para países em desenvolvimento, cerca de $15 \%$ da população brasileira é composta de pessoas com deficiência, sendo $50 \%$ com deficiência intelectual, $20 \%$ com deficiência física, 15\% com deficiência auditiva, 10\% com múltipla deficiência e 5\% com deficiência visual. No Brasil, conforme o Censo $\operatorname{IBGE}^{5}$ (2010), 23,9\% da população apresenta alguma deficiência, ou seja, aproximadamente 45 milhões de brasileiros, dos quais aproximadamente 1.250 são considerados surdo-cegos.

Sabe-se do grande desafio de manter uma comunicação com surdo-cegos. Todavia, alguns questionamentos surgem: há cirurgiões-dentistas capacitados? Como fazem os atendimentos?

Assim, este estudo, baseado em revisão de literatura, tem como objetivos: descrever conceitos, causas e classificações da surdo-cegueira; relatar os meios de comunicação com os surdo-cegos; discorrer sobre a necessidade da inserção do odontólogo na equipe multiprofissional de atendimento aos surdo-cegos e definir metodologias que auxiliem nesse atendimento.

\section{Surdo-cegueira}

A surdo-cegueira classifica-se em dois tipos: quanto ao período de aquisição da linguagem e quanto ao período de surgimento ${ }^{6}$. Com relação ao primeiro tipo, pode ocorrer em dois períodos distintos: antes da aquisição de uma linguagem (surdo-cegos pré-linguísticos ou pré-simbólicos) e após a aquisição da linguagem (surdo-cegos pós-linguísticos ou pós-simbólicos) $)^{2,7}$. As crianças surdo-cegas pré-linguísticas apresentam o olfato e o tato como os maiores meios de interação com o mundo. Essas limitações sensoriais tornam o desenvolvimento da comunicação bastante prejudicado ${ }^{8}$. Já os surdo-cegos pós-linguísticos são educados totalmente no abstrato e nos tipos de "resíduos" de comunicação que apresentam, que podem ser aproveitados e adaptados, a fim de lhes proporcionar maior autonomia ${ }^{9}$.

Quanto ao período de surgimento, classifica-se em: congênita ou adquirida ${ }^{6}$. A maior causa de surdo-cegueira é a rubéola congênita (40\% dos casos), tendo outras causas em menores proporções, a citar: microcefalia, síndrome de Goldenhar, síndrome de Lennox Gastaut, septicemia, eritroblastose fetal, prematuridade, meningite, síndrome de Usher, síndrome em estudo e causas desconhecidas. Convém lembrar que a rubéola congênita destaca-se como a maior etiologia de surdo-cegos pré-linguísticos ${ }^{6,10}$.

Por seu turno, o maior número de surdo-cegos pós-linguísticos corresponde àqueles que nasceram surdos e mais tarde perderam a visão, sendo a Síndrome de Usher a causa mais prevalente ${ }^{4}$.

Infelizmente, ainda existe uma falha nas informações aos pais e/ou responsáveis de pessoas surdo-cegas, devido à falta de conhecimento dos serviços de saúde e educação para orientá-los e encaminhá-los às instituições competentes. Diante disso, o surdo-cego pode sofrer as consequências de atraso no seu desenvolvimento ${ }^{11}$.

\section{Sentidos da visão e da audição na comunicação dos surdo-cegos}

Os sentidos são representados pela visão, pelo paladar, pela audição, pelo tato e pelo olfato. Quando a pessoa não apresenta um ou mais desses sentidos, ou mesmo quando estes estão diminuídos, os outros tendem a estar mais aguçados, com intuito de compensar a deficiência ${ }^{12}$.

A visão e a audição têm extrema importância no desenvolvimento da comunicação da criança. A visão permite que a criança tenha conhecimento do mundo a sua volta, bem como curiosidade em querer tocar os objetos. A audição favorece a percepção fora do campo visual, ou seja, permite reconhecer alguma situação sem que seja necessário ter proximidade em relação ao fato. A combinação desses dois sentidos favorece a capacidade de conhecer e experimentar a realidade, assim como antecipar os acontecimentos e prevenir possíveis perigos ${ }^{6,13,14}$.

Bebês surdo-cegos são erroneamente diagnosticados como tendo paralisia cerebral, o que se explica pelo fato de as dificuldades sensoriais impedirem o desenvolvimento psicomotor. Confusão de diagnósticos leva a que, muitas vezes, os pacientes sejam tratados como portadores de patologias como autismo (devido aos movimentos estereotipados) e encefalopatia crônica da infância ${ }^{15,16}$. Essa associação da surdo-cegueira com o autismo foi relatada na pesquisa de Wing e Wing ${ }^{17}$ (1971), que, ao investigarem 15 crianças com surdez e cegueira em níveis parciais, constataram que todas apresentavam comportamento semelhante ao de crianças autistas. Dentre essas crianças, 12 haviam herdado a deficiência das mães com rubéola na gestação. Os autores verificaram, ainda, que, apesar da grande dificuldade de comunicação e relacionamento do surdo-cego, os autistas possuíam maior severidade de interação social. 


\section{Comunicação e estreitamento dos laços profissional-paciente}

A comunicação resume-se na troca de uma mensagem entre duas pessoas ou mais, na qual uma a envia e a(s) outra(s) a recebe(m). Para a emissão, pode ser utilizada a fala, assim como o contato visual, expressões visuais, gestos e movimentos corporais; e o recebimento pode se dar escutando, observando e sentindo 4 .

Os trabalhos a serem desenvolvidos com os surdo-cegos devem direcionar-se segundo os seus próprios interesses. Com o surdo-cego pré-linguístico parte-se do princípio de investigar, conhecer o que mais lhe desperta atenção, sendo objetos ou até mesmo atividades, e a partir disso ter um direcionamento na conduta de trabalho. Enquanto o surdo-cego pós-linguístico, considerado na maioria das vezes mais passível de alterações. Deve-se respeitar o comportamento e desenvolvimento de cada indivíduo, pois cada um possui suas limitações ${ }^{18}$.

Existem alguns meios de comunicação com pessoas surdo-cegas, tais como: intérprete de Libras e guia-intérprete, Closet Circuit TV (CCTV), Braille, tellethouch, tablitas de comunicação, diálogos (fala escrita), alfabeto dactilológico, letras de forma, tadoma e sistema pictográfico ${ }^{4}$.

Certo de que não há uma regra para o tipo de comunicação a ser estabelecida com cada paciente, o profissional deverá buscar o melhor meio, a fim de obter sucesso no estabelecimento do vínculo.

\section{Surdo-cegos e humanização do atendimento}

O número de profissionais capacitados para o atendimento de pacientes com surdo-cegueira em instituições públicas ou privadas é muito reduzido ${ }^{19}$. Quando se pensa no atendimento às pessoas com necessidades especiais nos serviços de saúde, surge uma grande preocupação com relação à inclusão social, fato explicado pelo despreparo na comunicação dos profissionais, impedindo um atendimento humanizado e, consequentemente, desqualificando o serviço prestado à população $0^{20}$.

É de fundamental importância a inclusão da disciplina de pacientes com necessidades especiais nos currículos de graduação. No entanto, apenas 56,36\% das Faculdades de Odontologia no Brasil que responderam aos questionários confirmaram realizar a abordagem de assuntos relacionados a esses pacientes nos currículos de graduação ${ }^{21}$.

\section{Atuação do cirurgião-dentista em equipe multiprofissional}

$\mathrm{Na}$ literatura, percebe-se a falta de trabalhos com crianças surdo-cegas na área odontológica. Por que o cirurgião-dentista normalmente não está incluído na equipe multiprofissional de atenção ao indivíduo surdo-cego?

Esse profissional tem uma tarefa muito importante, uma vez que deve partir dele a intenção de comunicar-se, encontrando alternativas para essa aproximação ${ }^{22}$. Em vista disso, o cirurgião-dentista necessita de maiores informações fora de sua área de atuação, a fim de adquirir conhecimentos indispensáveis para relacionar e solucionar os problemas de ordem local e sistêmicos ${ }^{23}$.

Procedimentos odontológicos com anestesia, assim como consultas e exames médicos de maior complexidade, são alguns dos fatores que mais geram preocupações nas famílias ${ }^{10}$.

Por meio dessa revisão bibliográfica, propõe-se, por fim, um esquema prático para o atendimento odontológico do paciente surdo-cego. Não é objetivo, nem pretensão ditar regras, uma vez que cada paciente tem sua particularidade, servindo, portanto, como um auxílio no manejo da pessoa com surdo-cegueira.

\section{Cuidados no atendimento odontológico}

Inicialmente, é necessário realizar anamnese detalhada com os pais/responsáveis ou guia-interprete sobre as causas da surdo-cegueira ${ }^{24,25}$.

Existe uma relação entre surdez e hipoplasia dental, devido ao fato de o desenvolvimento do nervo auditivo ocorrer no período embrionário do dente, podendo provocar alterações na estrutura dentária ${ }^{26}$. Com relação aos deficientes visuais, não existe qualquer característica odontológica associada, apenas se percebe falta de higiene bucal, traumas dentais e lesões em tecidos moles por acidentes ou quedas ${ }^{27}$.

$\mathrm{Na}$ anamnese, é importante avaliar:

a) Grau de deficiência auditiva e visual, porque, dependendo do grau, podem variar o nível de comprometimento neuropsicomotor e agravos no seu desenvolvimento ${ }^{28}$. É fundamental que os pais informem o modo de comunicação mais adequado, seus objetos de referência e seus hábitos ${ }^{29}$

b) Como é feita a higiene bucal do paciente?

c) Orientar os pais/responsáveis o quanto é importante para o sucesso do tratamento a assiduidade nas consultas. 
Na primeira consulta, após certificar-se das informações prestadas pelos pais/responsáveis, o profissional deve observar o que desperta atenção na criança, suas atitudes e seus medos. Surdo-cegos costumam reconhecer as pessoas na maioria das vezes pelo olfato. Portanto, recomenda-se manter a mesma fragrância do perfume nas consultas. Outro sentido aguçado é o tato. Desse modo, caso algum objeto no consultório agrade ao paciente, é importante tê-lo sempre à disposição ${ }^{16}$.

Outra estratégia é procurar manter uma rotina de atendimento, para que o paciente possa memorizá-la, sentindo-se seguro naquele ambiente. Os pais sempre devem ser previamente informados a respeito do procedimento, e os auxiliares de saúde bucal devem estar capacitados, a fim de favorecer o sucesso do tratamento $^{30}$.

Iniciar por procedimentos mais simples (profilaxia e escovação), como adequação, ou seja, dessensibilização da cavidade bucal, é uma boa alternativa. Além disso, as consultas devem ser curtas, evitando que o paciente mantenha a boca aberta por muito tempo ${ }^{31}$.

É igualmente importante permitir que o paciente toque nos equipamentos e instrumentos a serem utilizados, oportunizando-lhe conhecer e sentir a textura, o tamanho, o peso e a temperatura. Se houver a possibilidade de optar entre materiais, o ideal é escolher aqueles que possuam menos odor e sabor mais agradável. Deve-se, ainda, evitar, ao máximo, movimentos bruscos e utilização de instrumentos que liberem resíduos (como água e pó) para não assustar o paciente ${ }^{32}$.

No caso de pacientes que utilizam aparelhos auditivos, sugere-se que estes sejam removidos, pois os sons de alta-rotação, ultrassom e sugadores poderão gerar desconforto durante 0 atendimento ${ }^{27}$.

Caso o paciente não seja colaborativo, pode-se optar por utilizar medicamentos, sob orientação médica, para reduzir a ansiedade, a fim de obter comportamento mais adequado, facilitando o atendimento. $\mathrm{O}$ cirurgião-dentista pode, ainda, utilizar recursos para estabilização do paciente (auxílio do acompanhante, de faixas, lençóis, estabilizadores) e dispositivos para manter a abertura bucal (abridores de silicone, borracha, madeira, plástico etc.). Como última opção, o paciente é levado a realizar os procedimentos odontológicos sob anestesia geral ${ }^{33}$.

Vale ressaltar que a garantia do sucesso no atendimento ao paciente surdo-cego dependerá da habilidade e aptidão do cirurgião-dentista, além do estabelecimento de um processo de mútua confiança entre ambos.

\section{Considerações finais}

Esta revisão de literatura evidencia uma precariedade de pesquisas a respeito do atendimento odontológico do paciente com surdo-cegueira, revelando a necessidade de mais pesquisas nessa área. Além disso, faltam recursos humanos capacitados tanto no serviço público como no privado, o que demonstra a necessidade de formar-se profissionais que saibam comunicar-se e atender adequadamente pessoas surdo-cegas.

É igualmente importante a implementação de políticas públicas que sirvam como respaldo legal para os surdo-cegos, evitando que continuem em situação de "exclusão social". Nesse sentido, tornam-se fundamentais a conscientização e a preparação dos pais/responsáveis quanto aos cuidados da pessoa com surdo-cegueira, uma vez que ainda existem aqueles que são mantidos "aprisionados" no lar por vergonha e não aceitação.

É de extrema relevância, para o sucesso do tratamento, que os profissionais da área conheçam e coloquem em prática os diferentes meios de comunicação, a fim de que possam escolher o mais adequado para cada paciente surdo-cego.

Por sua vez, a família tem um papel determinante para a evolução do tratamento da criança surdo-cega, pois servirá como um elo entre ela e o profissional, auxiliando na prestação de informações sobre sua rotina, seus costumes e os meios de comunicações mais apropriados.

O cirurgião-dentista, quando inserido na equipe multiprofissional, proporciona melhores condições de saúde e desenvolvimento do paciente com surdo-cegueira. A atenção ao paciente desde bebê até a fase adulta permite que o profissional crie vínculos e gere maior segurança e confiança durante $o$ atendimento.

Enfim, o profissional de odontologia, além de ter um amplo conhecimento técnico e científico, necessita conhecer um meio de comunicação efetivo com os pacientes surdo-cegos, contribuindo para que essa população obtenha melhores condições de saúde bucal.

\section{Abstract}

Introduction: dentistry has a very important role in the health of deafblind patients, yet it is hindered by the lack of trained professionals and specific studies in this area. Objective: this literature review, aims to present to dental professionals information on deafblindness describing concepts, causes, and classifications, as well as to report communication methods, the need for dentist insertion in the multidisciplinary care team, and to define strategies to assist dental care of these patients. Literature review: the work of the dentist as part of a multidisciplinary teamwork provides patients with improvements in their development by creating bonds and activities leading to greater autonomy. This study also shows the lack of information in the field of deafblindness, revealing that the inclusion of patient's family is a fundamental factor for a favorable prognosis on educational, social, and communication processes. Final remarks: in addition to technical and scientific knowledge in the field, the dentist should know the best way to communicate with the deafblind patient.

Keywords: Deafblind. Interdisciplinary communication. Disabled persons. Communication barriers. 


\section{Referências}

1. Conselho Federal de Odontologia. Resolução 22/2001, de 27 de janeiro de 2001. Normas sobre anúncio e exercício das especialidades odontológicas e sobre cursos de especialização [online]; 2001 [citado 2013 Jan. 14]. Disponível em URL: http://www.cfo.org.br.

2. Cader-Nascimento FAAA, Costa MPR. Mediação pedagógica no processo de desenvolvimento da comunicação em crianças surdocegas. Temas em Psicologia da SBP 2003; 11(2):85-96.

3. Lagati S. "Deaf-Blind" or "Deafblind"? International perspectives on terminology. Journal of Visual Impairment and Blindness 1995; 89(3):306.

4. Garcia A. Surdocegueira: empírica e científica. São Luis Gonzaga: [s.n]; 2008

5. Instituto Brasileiro de Geografia e Estatística (IBGE) [online] [citado 2012 fev. 02]. Disponível em URL: http://www. ibge.gov.br.

6. Santos PC, Gallo MTA. Crianças com surdocegueira congênita atendidas em um centro referência de Salvador-BA: sete casos, Nossos Meios, RBC [periódico online]. [citado 2011 ago. 15]; 41(3). Disponível em URL: http://www.ibc.gov. $\mathrm{br} /$ ?catid $=4 \&$ itemid $=10162$.

7. Kinney R. A definição, responsabilidades e direitos dos surdocegos. In: I Seminário Brasileiro de Educação do deficiente Audiovisual - ABEDEV: 1977; São Paulo. Anais; 1977

8. Bove M, Riggio M. La comunicación pre-linguistica com enfasis em la discapacidad severa. In: XI Conferencia Internacional para a educação dos surdocegos, IADB: 1995; Córdoba, Argentina. Resumes; 1995. p. 27.

9. Garcia A. AGAPASM estimula surdocegos e multideficientes a superar limites. PortoWeb. [online] 2007 [citado 2012 abr. 21]; Disponível em URL: http://www1.prefpoa.com.br/pwcidadao/default.php?reg=14\&p_secao=143.

10. Aráoz SMM. Experiências de pais de múltiplos deficientes sensoriais: surdocegos: do diagnóstico à educação especial [Dissertação de Mestrado]. São Bernardo do Campo: Universidade Metodista de São Paulo/UMESP; 1999.

11. Arias MH, Zeferino AMB, Filho AAB. Características clínico-sociais do surdocego institucionalizado. Rev Paul Pediatria 2006; 24(1):20-6.

12. Möller CC, Ibaldo LT, Tovo MF. Avaliação das condições de saúde bucal de escolares deficientes auditivos no município de Porto Alegre, RS, Brasil. Pesq Bras Odontop Clin Integ $2010 ; 10(2): 195-200$.

13. Amaral I. Comunicação com crianças surdocegas. Caderno de Educação Infantil 1997; (47/97):8-11.

14. Maia SR. Confronto entre a criança surdocega e o mundo exterior. Projeto Horizonte [on line] 2006 [citado 2010 jun. 26]; Disponível em URL: http://www.ahimsa.org.br.

15. Leuw L. Diagnóstico, avaliacão e planejamento educacional da criança surda-cega. In: I Seminário Brasileiro de Educação do Deficiente Audiovisual, ABEDEV: 1977; São Paulo. Anais; 1977. p. 59-72

16. Maia SR, Aráoz SMM. A surdocegueira - "Saindo do Escuro". Revista Educação Especial: Cadernos de Educação Especial / Universidade Federal de Santa Maria. Centro de Educação / Departamento de Educação Especial / Laboratório de Pesquisa e Documentação - LAPEDOC 2001; 1(17):90.

17. Wing L, Wing JK. Multiple impairments in early childhood autism. Journal Autism Child Schizophr 1971; 1(3):256-66.

18. Chen D, Dote-Kwan J. Starting points: instructional pratices for young children whos multiple desabilities include visual impairment. Los Angeles: Blind Children Center; 1995.

19. Ferioli G, Bove ME, Perreault S. Formación en América Latina: puente al milênio. In: IV Conferencia Europea DbI ed. ONCE: 1997; Madrid. Actas; 1997. p. 39.

20. Chaveiro N, Barbosa MA. Assistência ao surdo na área de saúde como fator de inclusão social. Rev Esc Enferm USP 2005; 39(4):417-22.

21. Fassina AP, Crosato E, Júnior JSM. Presenca da disciplina e/ou conteúdo de Pacientes Portadores de Necessidades Especiais nas Faculdades de Odontologia no Brasil em 2005. Odontologia e Sociedade 2007; 9(2):1-4

22. Varellis MLZ. Alterações sensoriais. In: Varellis MLZ. O paciente com necessidades especiais na odontologia. São Paulo: Santos; 2006. p. 367-76.

23. Araújo NCB. Proposta para reconhecimento da especialidade Pacientes Especiais aprovada na assembléia preparatória estadual da ANEO de MT. [online] 2001 [citado 2009 jan. 14]. Disponível em URL: http://www.odontologia.com.br/artigos.

24. Silva OMP, Panhoca L, Blachman IT. Os pacientes portadores de necessidades especiais: revisando os conceitos de incapacidade, deficiência e desvantagem. Salusvita. 2004 23(1):109-16

25. Marta SN. Programa de assistência odontológica ao paciente especial: uma experiência de 13 anos. RGO 2011 Jul-Set; 59(3):379-85

26. Miller JB. Dental care for deaf child. J. Ocklahoma State Dent 1970; 60(3):38-43.

27. Haddad AS, Ferreira MCD. Deficiências sensoriais e de comunicação. In: Haddad AS. Odontologia para pacientes com necessidades especiais. São Paulo: Santos 2007. p. 253-61.

28. Souza TA, Souza VE, Lopes MCB, Kitadai SPS. Descrição do desenvolvimento neuropsicomotor e visual de crianças com deficiência visual. Arq Bras Oftalmol 2010; 73(6):526-30.

29. Boas DCV, Ferreira LP, Moura MC, Maia SR. A comunicação de pessoas com surdocegueira e a atuação fonoaudiológica. Distúrb Comum 2012; 24(3):407-14.

30. Aráoz SMM, Costa MPR. Aspectos biopsicossociais na surdocegueira. Rev Bras Ed Esp 2008 Jan-Abr; 14(1):21-34

31. Puesta AL. Intervención odontológica actual en niños con autismo. La desensibilización sistemática. Cient Dent 2009; 6(3):207-15.

32. Carvalho ACP, Figueira LCG, Utumi ER, Oliveira CO, Silva, LPN. Pedron IG. Considerações no tratamento odontológico e periodontal do paciente deficiente visual. Rev Odontol Bras Central 2010; 19(49):97-100.

33. Peres AS, Peres SHCS, Silva RHA. Atendimento a pacientes especiais: reflexão sobre os aspectos éticos e legais. Rev Fac Odontol Lins 2005; 17(1):49-53.

Endereço para correspondência:

Antônio Augusto Iponema Costa

Rua Alberto Zanatta, 527, apto 406, Bairro

Loteamento Bosque

99150-000 - Marau - RS

Fone: (54) 9646-8785

E-mail: antonioiponema@ig.com.b

Recebido: 11/03/2013. Aceito: 16/07/2013. 